\title{
Care of Patients Who Are Worried about Mercury Poisoning from Dental Fillings
}

\author{
David Vearrier, MD, and Michael I. Greenberg, MD, MPH
}

Introduction: Public concern about adverse health effects from mercury exposure from dental amalgams remains a high-profile issue. Patients with nonspecific neuropsychiatric symptoms may incorrectly attribute their complaints to mercury poisoning, and some alternative medical providers diagnose mercury toxicity using nonvalidated tests or without testing at all.

Case report: We report the case of a 37-year-old female who was referred to our outpatient medical toxicology clinic by her family medicine physician after a wellness doctor involved in her care ordered a dimercaptopropanesulfonic acid (DMPS) challenge urine study that revealed an "elevated" mercury level.

Discussion: The use of postchelator challenge urine testing to diagnose mercury poisoning has not been validated. Use of such tests may cause falsely elevated urine mercury levels resulting in misdiagnosis of mercury poisoning and unncessary, expensive, and potentially dangerous chelation therapy.

Conclusion: Family medicine physicians may encounter patients who are concerned about mercury poisoning after undergoing postchelator challenge urine testing. In patients with a low suspicion for mercury toxicity, reassurance is adequate. In patients with moderate to high suspicion for mercury toxicity, a validated test for mercury, such as a 24-hour urine mercury level, or referral to a medical toxicologist is the most appropriate approach. ( $\mathrm{J}$ Am Board Fam Med 2010;23:797-798.)

Keywords: Heavy Metals, Mercury, Mercury Poisoning, Chelation Therapy

Public concern about mercury toxicity from dental amalgams remains an issue today despite the bulk of the scientific evidence suggesting that mercury amalgams do not result in adverse health effects. ${ }^{1-6}$ Mercury toxicity may result in a number of nonspecific neuropsychiatric symptoms, and patients who research their complaints using lay books, magazines, or the Internet may incorrectly ascribe their complaints to mercury toxicity. Some alternative medical providers may diagnose mercury toxicity using nonvalidated tests. We operate a medical toxicology clinic staffed by board-certified medical toxicologists where we see patients who have been referred by primary care physicians and

This article was externally peer reviewed.

Submitted 19 February 2010; revised 17 June 2010; accepted 23 June 2010.

From the Department of Emergency Medicine, Drexel

University College of Medicine, Philadelphia, PA.

Funding: none.

Conflict of interest: none declared.

Corresponding author: David Vearrier, MD, Department of Emergency Medicine, Albert Einstein Medical Center, Korman B-6, 5501 Old York Road, Philadelphia, PA 19141 (E-mail: vearried@einstein.edu). occupational physicians because of concern about toxic exposures. We recently saw a patient who was referred to our clinic by her family physician after she reportedly had elevated urine mercury levels on a chelator challenge urine test that had been ordered by her "wellness physician."

The patient, a 37-year-old woman, complained of fluctuations in weight, fatigue, weakness, agitation, difficulty with memory, numbness in her fingertips, and hair, skin, and nail changes during the preceding 4 years, which she was concerned were caused by her dental amalgams. Her medical history included hypothyroidism alternately treated with levothyroxine and porcine thyroid powder by her endocrinologist and her wellness physician, respectively. Her occupational history was negative for any remote or recent heavy metal exposure. Her physical examination was normal.

A dimercaptopropanesulfonic acid (DMPS) challenge urine study ordered by the patient's wellness physician reported an elevated urine mercury level of $53 \mu \mathrm{g}$ of mercury per gram of creatinine. No reference range has been established for this test. Subsequently, a 24-hour urine mercury level was ordered 
by her family physician on our advice and contained $<4 \mu \mathrm{g}$ of mercury per liter of urine (normal, $<20 \mu \mathrm{g}$ of mercury per liter of urine).

The use of a chelator challenge urine test for heavy metals is a nonvalidated test for the diagnosis of heavy metal toxicity. Also known as postchallenge or postprovocation urine testing, the patient receives a chelating agent such as dimercaptosuccinic acid, dimercaprol, edetate calcium disodium, or DMPS. Depending on the agent used, the dose and route (oral or intravenous) of the chelator varies. In our patient's case, DMPS $250 \mathrm{mg}$ was administered intravenously. Urine is then collected for a specified period of time after administration of the chelator. In the case of our patient, urine was collected for 2 hours afer DMPS administration. The use of a chelating agent increases the urinary concentrations of heavy metals. ${ }^{7}$ This results in falsely elevated levels of urine heavy metals, including mercury. Normal reference ranges have not been established for postchelator challenge urine testing for heavy metals. ${ }^{8}$

The increase in use of postchelator challenge urine testing by alternative medical providers has led the American College of Medical Toxicologists to issue a position statement recommending against the use of such testing, stating that "[it] is...the position of the American College of Medical Toxicology that postchallenge urinary metal testing has not been scientifically validated, has no demonstrated benefit, and may be harmful when applied in the assessment and treatment of patients in whom there is concern for metal poisoning." There are no laws, however, protecting patients from non-evidence-based medicine or preventing the use of nonvalidated testing procedures.

The most important tool in the evaluation of a patient who is concerned about mercury poisoning is a careful occupational and environmental history, including possible dietary exposure through the consumption of seafood. In patients for whom there is concern of mercury exposure based on history or physical examination, a 24-hour urine collection is the most appropriate test to evaluate for inorganic or elemental mercury exposure, whereas a whole-blood mercury level is most useful for methylmercury exposure. ${ }^{5}$ These tests are typically available through regional reference laboratories.

In our patient, some or all her symptoms may have been caused by alternating use of levothyroxine and porcine thyroid powder for control of her hypothyroidism. We did not, however, review the results of her thyroid function tests. Our recommendations included no further chelation therapy, no further testing for heavy metals, the discontinuation of porcine thyroid powder, and further management of her hypothyroidism, as per her boardcertified endocrinologist.

Family medicine physicians and other physicians involved in the primary care of patients may encounter the issue of postchelator challenge urine testing. The use of chelator challenge urine testing is not validated for the diagnosis of heavy metal toxicity and may result in patients being encouraged to undergo expensive, unnecessary, and potentially dangerous chelation therapy. Patients with a low suspicion for heavy metal toxicity and positive postchelator challenge urine testing for heavy metals often only require reassurance. For patients with moderate to high suspicion for heavy metal toxicity, such as those with occupational exposure to mercury, the use of a validated test for heavy metals or referral to a medical toxicologist is the most appropriate approach.

\section{References}

1. Bellinger DC, Trachtenberg F, Barregard L, et al. Neuropsychological and renal effects of dental amalgam in children: a randomized clinical trial. JAMA 2006;295:1775-83.

2. DeRouen TA, Martin MD, Leroux BG, et al. Neurobehavioral effects of dental amalgam in children: a randomized clinical trial. JAMA 2006;295:1784-92.

3. Vamnes JS, Eide R, Isrenn R, Hol PJ, Gjerdet NR. Blood mercury following DMPS administration to subjects with and without dental amalgam. Sci Total Environ 2003;308:63-71.

4. Schuurs A, Exterkate R, ten Cate JM. Biological mercury measurements before and after administration of a chelator (DMPS) and subjective symptoms allegedly due to amalgam. Eur J Oral Sci 2000;108:511-22.

5. Kales SN, Goldman RH. Mercury exposure: current concepts, controversies, and a clinic's experience. J Occup Environ Med 2002;44:143-54.

6. Bailer J, Rist F, Rudolf A, et al. Adverse health effects related to mercury exposure from dental amalgam fillings: toxicological or psychological causes? Psychol Med 2001;31:255-63.

7. Torres-Alanis O, Garza-Ocanas L, Bernal MA, Pineyro-Lopez A. Urinary excretion of trace elements in humans after sodium 2,3-dimercaptopropane-1-sulfonate challenge test. J Toxicol Clin Toxicol 2000;38:697-700.

8. Charlton N, Wallace KL. American College of Medical Toxicology position statement on post-chelator challenge urinary metal testing. Available at: http://www.acmt.net/ cgi/page.coi? aid $=2999 \& \_i d=52 \&$ zine $=$ show. Accessed 24 November 2009. 